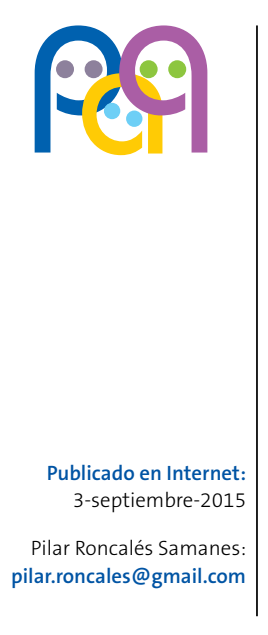

\title{
Original
}

\section{Recogida de orina en el lactante febril para el diagnóstico de la infección urinaria en Urgencias}

\author{
M. P. Roncalés Samanes, P. Caudevilla Lafuente, E. Sancho Gracia, V. Gómez Barrena, \\ R. Pérez Delgado, C. Campos Calleja
}

Servicio de Pediatría. Hospital Universitario Miguel Servet. Zaragoza. España.

Introducción: la infección del tracto urinario (ITU) es una enfermedad frecuente en niños. Resulta fundamental una adecuada recogida de orina para evitar falsos positivos, minimizando procedimientos invasivos. La recogida de orina al acecho es una técnica incruenta, con baja tasa de contaminación, por lo que se estableció como método de elección al actualizar nuestro protocolo clínico.

Material y métodos: estudio prospectivo longitudinal descriptivo y analítico, realizado en el Servicio de Urgencias de un hospital terciario, en dos periodos, antes y después de la actualización del protocolo. Se analizaron: edad, sexo, tiempo en Urgencias, método de recogida, sedimento y cultivo de orina y diagnóstico, en pacientes febriles menores de tres años.

Resultados: hubo 140 pacientes en 2012 y 180 en 2011, sin diferencias en distribución por sexo y edad. Las medias etarias fueron 12 y 14 meses respectivamente. El 35,7\% se recogieron por acecho en 2012, frente al 7,8\% de 2011 ( $p<0,001$ ). En 2011 fueron necesarias más confirmaciones: 20,5\% frente a $10,7 \%$ en 2012. No hubo diferencias significativas en el número de sospechas de ITU ni en el de muestras contaminadas en ambos años. Tampoco entre las muestras recogidas por acecho o sondaje en 2012. El tiempo medio de estancia en Urgencias en 2011 fue 221 minutos, siendo 190 en 2012 ( $p<0,05)$.

Palabras clave: Conclusiones: la recogida de orina mediante el acecho constituye un método sencillo y no invasivo, que generalmente no precisa confirmación, por lo que reduce el tiempo de espera en el Servicio de Urgencias. No se ha objetivado un aumento de falsos diagnósticos de ITU en pacientes con muestra recogida por acecho.

\section{Collection of urine in febrile infants for diagnosis of urinary tract infection in the Emergencies' room}

- Lactante

- Urgencias de Pediatría

Introduction: urinary tract infection (UTI) in children is a common process. It is essential to find an adequate method to collect urine, to avoid false positives, minimizing invasive procedures. Clean catch urine (CCU) is a noninvasive technique, with low contamination rate, so it has been established as the recommended method for urine collection to update our clinical protocol.

Material and methods: a prospective longitudinal descriptive and analytical study was conducted in a tertiary hospital in the Emergencies' room (ER) in two periods, before and after the update protocol. Age, sex, time in the ER, collection method, sediment and urine culture and diagnosis in febrile patients $<3$ years were analyzed.

Results: there were 140 patients in 2012 and 180 in 2011, with no differences in age and sex distribution. The mean ages were 12 and 14 months respectively. $35.7 \%$ of the samples were collected by CCU in 2012 , compared to $7.8 \%$ in $2011(p<0.001)$. In 2011 more confirmations of urine analysis were necessary: $20.5 \%$ vs. $10.7 \%$ in 2012 . There were no significant differences between the number of suspected UTI or the contaminated samples in both years. Neither did between samples collected by catheterization or CCU in 2012. The average length of stay in the ER in 2011 was 221 minutes, while 190 in $2012(p<0.05)$.

Key words:

- Urine collection

method

- Children

- Clean catch urine

- Emergency room

Conclusions: urine collection by CCU is a simple and noninvasive method, which usually does not require confirmation, thereby reducing the wait time in the ER. There has not been an objectified increased of false diagnosis of UTI in patients with sample collected by CCU.

Cómo citar este artículo: Roncalés Samanes MP, Caudevilla Lafuente P, Sancho Gracia E, Gómez Barrena V, Pérez Delgado R, Campos Calleja C. Recogida de orina en el lactante febril para el diagnóstico de la infección urinaria en Urgencias. Rev Pediatr Aten Primaria. 2015;17:205-11. 


\section{INTRODUCCIÓN}

La orina es habitualmente estéril. La infección del tracto urinario (ITU) es una enfermedad frecuente en la edad pediátrica que viene definida por la existencia de bacterias en el tracto urinario en presencia de síntomas compatibles ${ }^{1}$.

Hablamos de ITU cuando existe una sintomatología clínica asociada a un recuento bacteriano en orina significativo según el procedimiento de recogida de la muestra de orina para el urocultivo. Existe una gran variabilidad clínica en esta patología, que depende en gran medida de la edad de presentación y la localización de la ITU ${ }^{1}$.

Resulta fundamental una adecuada recogida de la muestra de orina para evitar un falso positivo, utilizando el método más estéril posible, evitando procedimientos invasivos.

La mejor técnica para obtener muestras de orina no contaminadas es la aspiración directa de la vejiga mediante punción suprapúbica ya que evita el paso de la orina por la uretra. Se trata de un procedimiento sencillo, aunque invasivo y con una variable tasa de éxito (23-90\%). En segundo lugar se encuentra el sondaje vesical con una sensibilidad de hasta $95 \%$ y especificidad de $99 \%$. Sin embargo se debe tener en cuenta el efecto psicológico en mayores de un año e incluso la necesidad de sedación en alguna ocasión. Se dispone de otros métodos de recogida como la bolsa adhesiva y el chorro medio de la micción ${ }^{2}$.

Según las últimas guías de práctica clínica ${ }^{1}$ la recogida mediante bolsa adhesiva presenta un mayor índice de contaminación, en comparación con otros métodos, como el sondaje vesical o la punción suprapúbica. Sin embargo, se debe encontrar el método de recogida menos invasivo para los pacientes y simultáneamente con menor riesgo de contaminación.

La recogida del chorro medio de la micción está aceptado como método adecuado para la recogida de muestras de orina en niños continentes. La recogida al acecho es equivalente en niños sin capacidad de control de esfínteres.
En 2011 se llevó a cabo una actualización de nuestro protocolo de Urgencias, en la que, entre otras medidas, se introdujo la recogida al acecho como técnica de elección en la recogida de muestras de orina en niños no continentes.

\section{MATERIAL Y MÉTODOS}

Se ha llevado a cabo un estudio prospectivo longitudinal descriptivo y analítico, realizado en un hospital terciario (Hospital Universitario Infantil Miguel Servet, Zaragoza, España), en el periodo comprendido entre el 12 de octubre y el 20 de diciembre de 2012. La población a estudio está compuesta por los pacientes menores de tres años con fiebre, a los que se realizó sedimento de orina, atendidos en la Unidad de Urgencias de nuestro hospital. Posteriormente se realizó la comparación de los resultados obtenidos con los del mismo periodo del año anterior.

Se realizó análisis de orina, según protocolo de nuestro hospital, a todo paciente menor de tres meses de edad, con fiebre mayor de $38^{\circ} \mathrm{C}$ sin focalidad aparente, $y$ a aquellos con edad comprendida entre 3 y 36 meses con fiebre sin foco mayor de $38,5^{\circ} \mathrm{C}$, o bien aquellos que presentaban fiebre mayor de $38^{\circ} \mathrm{C}$ y clínica miccional. Se han excluido aquellos casos con clínica miccional sin fiebre.

Consideramos alteración en el análisis básico de orina cuando hay nitritos positivos o leucocituria, distinguiendo en este caso, según el método de recogida de la muestra, como sigue: en muestras recogidas por bolsa, más de 20 leucocitos por campo $(\mathrm{L} / \mathrm{C})$ en varones y más de 50 en mujeres, siendo dudoso entre 10-20 y 30-50 respectivamente; en muestras recogidas al acecho, 10 leucocitos por campo independientemente del sexo; en las recogidas por sondaje más de 3-5 L/c.

En todos los casos se han recogido datos epidemiológicos (edad, sexo, antecedentes nefrourológicos), tiempo de estancia en urgencias y tiempo de recogida de la orina, método de recogida, resultados analíticos del básico de orina y urocultivo, diagnóstico final, necesidad o no de ingreso hospitalario y tratamiento al alta. 


\section{RESULTADOS}

Se obtuvieron un total de 140 muestras en 2012 (1,5\% del total de urgencias atendidas en este periodo en el hospital, y 180 en 2011 (1,3\% de los pacientes atendidos en el mismo centro en el periodo correspondiente).

En el año 2012 el 55\% de las muestras fueron varones y $45 \%$ mujeres, y en el año previo el porcentaje fue inverso. La media de edad fue de 12 meses en 2012 (rango de 36 días a 35,6 meses, mediana de 13 meses) y en 2011 la media fue de 14 meses (rango de 9 días a 35,5 meses, y mediana 11 meses). No se apreciaron diferencias significativas según la distribución por sexo ni edad en las muestras de 2011 y 2012.

Sí se han encontrado diferencias estadísticamente significativas con respecto al número de muestras de orina recogidas al acecho y por bolsa adhesiva en ambos periodos: 35,7\% en 2012 fueron por acecho o el chorro medio de la micción, frente al 7,8\% de 2011 ( $p<0,001$ ). Además en 2011 fue necesario un mayor número de confirmaciones, siendo esta diferencia significativa (20,5\% en 2011 y $10,7 \%$ en 2012), y estas en su mayor parte fueron por sondaje $(19,4 \%$ frente a $7,1 \%$, respectivamente) $(p<0,001)$.
En 2011 hubo 48 sospechas de ITU (26,6\%), 119 sindromes febriles sin foco $(66,1 \%)$ y 13 pacientes fueron catalogados de otros diagnósticos (7,2\%). En 2012, 36 pacientes fueron dados de alta de Urgencias con el diagnóstico de sospecha de ITU (25,7\%, porcentaje similar al año previo), 80 casos de síndrome febril sin foco $(57,14 \%)$ y 24 con otros diagnósticos (17,14\%). No se hallaron diferencias significativas en el número de sospechas de ITU en ambos años.

En la Tabla 1 se recogen los resultados de los urocultivos en los dos periodos. No se encontraron diferencias significativas con respecto al número de muestras contaminadas en los dos grupos. En 2012, año en el que hubo un mayor número de muestras recogidas mediante acecho, se compararon los resultados de los urocultivos recogidos mediante acecho con aquellos recogidos por sondaje vesical. Del total de sospechas de ITU en el grupo del acecho, el 30,1\% de los urocultivos fueron negativos y en el $7,6 \%$ se halló flora mixta, mientras que del grupo del sondaje el 38,4\% fueron negativos, sin encontrarse diferencias significativas en los dos grupos.

En relación con los antecedentes nefrourológicos, en 2011 seis pacientes habían presentado ITU previamente $(3,3 \%)$ y diez estaban diagnosticados de

\begin{tabular}{|c|c|c|c|c|c|c|c|c|c|}
\hline & & & & & & & & & \\
\hline Muestras o & & $140(1,5$ & el total de & rgencias) & & $180(1,3)$ & I total de & sencias) & \\
\hline Sexo & & Varones & o, hembra & $15 \%$ & & Varones & hembra & & \\
\hline Edad & Media & 12 mese & & & & 14 mese & & & \\
\hline & Rango & 36 días & meses & & & 9 días a & meses & & \\
\hline & Mediana & 13 mese & & & & 11 mese & & & \\
\hline Sospechas & & $36(25,7$ & & & & $49(27,2)$ & & & \\
\hline Recogida de & & Total & Acecho & Sonda & Bolsa & Total & Acecho & Sonda & Bolsa \\
\hline Urocultivo & Positivo & $21^{*}$ & 8 & 8 & 5 & $31^{* *}$ & 4 & 22 & 5 \\
\hline & Negativo & 9 & 4 & 5 & 0 & 11 & 1 & 10 & 0 \\
\hline & FM/cont. & 1 & 1 & 0 & 0 & 5 & 1 & 4 & 0 \\
\hline & Total & 31 & 13 & 13 & 5 & 47 & 6 & 36 & 5 \\
\hline & No rec. & 5 & & & & 2 & & & \\
\hline
\end{tabular}

${ }^{*}$ E. coli.

${ }^{* *}$ E. coli (25), E. faecalis (1), P. aeruginosa (4) y S. marscescens (1).

FM/cont.: flora mixta/contaminado; ITU: infección del tracto urinario; No rec.: no recogido/no consta. 
ectasia piélica (5,5\%). Cabe destacar que no todos los casos con estos antecedentes presentaron alteraciones en el sedimento de orina (75\% alterado, $25 \%$ normal). En 2012, siete pacientes habían sido diagnosticados en alguna ocasión de ITU (3,6\%), tres presentaban diagnóstico de ectasia $(2,1 \%)$ y cinco eran controlados por reflujo vesicoureteral (5,6\%). Tampoco en este periodo todos los que tenían antecedentes personales fueron finalmente ITU, presentando el $40 \%$ alteración en el sedimento de orina.

Con respecto a la estancia total en Urgencias, el tiempo medio en 2011 fue de 221 minutos, mientras que en 2012 fue 190 (siendo esta diferencia estadísticamente significativa, $p<0,05$ ). En cuanto al tiempo medio de espera en función del método de recogida, en 2012 fue de 73 minutos en los pacientes con bolsa adhesiva, frente a los 41 minutos en aquellos con recogida al acecho. En los casos recogidos mediante acecho/chorro miccional en 2011 el tiempo medio de espera fue de 63 minutos, sensiblemente inferior respecto a los que se recogió por bolsa (97 minutos).

En el año 2012 hubo significativamente menor número de ingresos en pacientes con ITU ( $p<0,05)$, $50 \%$, frente a $79,2 \%$ en 2011 , todo ello asociado a una modificación en los criterios de ingreso del protocolo diagnóstico-terapéutico de la ITU febril aprobado en nuestro hospital.

\section{DISCUSIÓN}

La ITU es una causa frecuente de fiebre en la infancia, representando hasta el 5-8\% de las fiebres de causa desconocida en lactantes ${ }^{3,4}$. En nuestra serie, el porcentaje de sospechas de ITU en estos casos fue de alrededor del 25\%, siendo los urocultivos positivos en 58,3\% en 2012 (14,9\% de ITU confirmadas en el total de pacientes de nuestra muestra) y 64,6\% en 2011 (17,2\%).

El retraso en el diagnóstico de una pielonefritis aguda puede dar lugar a lesiones en el parénquima renal, mientras que un diagnóstico erróneo puede originar conductas diagnósticas y terapéuticas inútiles, dolorosas, peligrosas y costosas para el individuo y la comunidad ${ }^{5}$.

El urocultivo es el gold standard para el diagnóstico de la ITU, pero lograr una muestra no contaminada es difícil en los niños pequeños. Los métodos de recogida de orina habitualmente utilizados son la bolsa adhesiva, el sondaje uretral, la punción suprapúbica y la recogida al acecho. Tanto el sondaje uretral como la punción suprapúbica se consideran las técnicas más recomendables para minimizar los falsos positivos, pero son más invasivas y dolorosas. Son técnicas cruentas, no exentas de riesgos, ya que pueden asociar dolor tras el procedimiento, lesiones de la vía urinaria y riesgo de infecciones secundarias a la realización de la técnica. La bolsa adhesiva es no invasiva y una alternativa de fácil utilización, pero se asocia a una alta tasa de contaminación $n^{6,7}$. Hernangómez et al. llevaron a cabo un estudio en 124 pacientes con síndrome febril o sospecha de ITU en el que analizaron las complicaciones en los pacientes sometidos a sondaje urinario puntual. Tras contactar con 116 pacientes del total, tres de ellos presentaron dolor a nivel de área genital, un caso hematuria no confirmada y un caso de ITU 12 días tras la cateterización vesical, concluyendo que este procedimiento es efectivo y seguro; sin embargo, se trata de una técnica invasora que necesita controles de calidad ${ }^{8}$.

En 2012, Karacan et al. realizaron un estudio con 1067 pacientes en los que se comparaban la bolsa adhesiva, el sondaje uretral, la punción suprapúbica y la recogida al acecho. Entre los resultados, destacaba una tasa de contaminación del acecho del $14,3 \%$, similar a la obtenida en las orinas recogidas mediante sondaje. En aquellas obtenidas por bolsa, la tasa de contaminación fue del $43,9 \%$ y en la punción suprapúbica del 9,1\%. En este estudio señalan que el método preferido fue la recogida al acecho, pero destacan la dificultad para llevarlo a $c^{c a b o}{ }^{9}$. En nuestra experiencia, se ha demostrado que si existe colaboración por parte tanto de la familia como del personal de la unidad, así como un lugar apropiado para su realización, el acecho es una técnica de escasa complejidad. Además, es la técnica menos agresiva para los pacientes. 
La mayoría de estudios que comparan métodos de recogida de orina no incluyen el chorro medio de la micción en niños no continentes. Un estudio realizado en el Institute of Maternal and Child Health of Pernambuco (IMIP, Recife, Brasil) compara muestras de orina recogidas en niños menores de 3 años, sin antecedentes de ITU. En este estudio, las muestras recogidas mediante el acecho presentaban contaminación en el $14,7 \%$ de los casos, siendo significativamente menor que en las recogidas mediante bolsa adhesiva $(26,6 \%)^{10}$. Otro estudio, que comparó los diferentes métodos de recogida de muestra de orina en niños menores de dos años, asocia la recogida mediante acecho con un índice de contaminación del $26 \%$, significativamente mayor que aquellas recogidas por sondaje (12\%) o punción suprapúbica (1\%). Relacionan el elevado porcentaje de contaminación en la primera técnica citada con la falta de estándares de recogida, y recomiendan nuevos estudios, dada la alta variabilidad de tasas de contaminación en la bibliografía ${ }^{11}$. En nuestra experiencia, las cifras de contaminación en las muestras recogidas mediante el acecho y aquellas por sondaje vesical fueron similares, por lo que podemos considerar el acecho como una técnica válida para la obtención de muestras de orina. Sin embargo, se han objetivado en nuestro estudio mayores tasas de contaminación que las descritas en la bibliografía, hasta un 42,8\%. Por este motivo, debería valorarse como criterio a mejorar, con el fin de optimizar la esterilidad en ambos procedimientos. Creemos que este hecho es debido a falta de información a las familias cuando se explica la técnica, por lo que se podría mejorar con el entrenamiento adecuado al personal sanitario.

Conforme a los protocolos de la Asociación Española de Pediatría de 2014, en los niños que no controlan su esfínter urinario, el método de recogida debe ser tanto más fiable (con menor riesgo de contaminación) cuanto más urgente sea establecer el diagnóstico e iniciar el tratamiento, y consideran que la recogida al acecho está aceptada como método de recogida fiable, aumentando su rentabilidad con maniobras previas de estimulación abdominal y lumbosacra ${ }^{12}$.
Según nuestro estudio, el acecho es un método para recoger orina que reduce el tiempo de espera con respecto a la bolsa adhesiva, aunque la técnica más rápida y fiable es el sondaje vesical. Sin embargo, se trata de un procedimiento no invasivo en comparación con el sondaje vesical y, en nuestra experiencia, los padres prefieren intentar la recogida mediante acecho y posponer y, si es posible, evitar la realización de un sondaje vesical.

En las Recomendaciones de la Conferencia de Consenso "Manejo Diagnóstico y Terapéutico de las Infecciones del Tracto Urinario en la Infancia" se nombra la media micción como técnica de elección e niños continentes, con un grado de recomendación B, pero no hacen referencia, sin embargo, a niños no continentes ${ }^{13}$.

La guía británica del National Institute for Health and Care Excellence (NICE) recomienda como método de recogida la micción limpia en todos los grupos de edad, tanto para análisis y sedimento, como para urocultivo ${ }^{14}$. En nuestra experiencia, el acecho en lactantes y niños menores de tres años supone un método equivalente al chorro medio de la micción en pacientes continentes.

Recientemente se ha publicado una nueva técnica para estimular la micción en niños incontinentes (en periodo neonatal) mediante la percusión de la zona suprapúbica y lumbar, con una tasa de éxito de hasta el $86,3 \%$ de los pacientes. El objetivo del estudio fue, además, la obtención de la muestra de orina en un periodo de tiempo inferior a los cinco minutos. La mediana fue de 45 segundos hasta la recogida de la muestra ${ }^{15}$.

En nuestro estudio, no se han encontrado diferencias significativas con respecto al número de contaminaciones en muestras recogidas mediante acecho frente al sondaje. Sin embargo, debemos tener en cuenta que de las 36 sospechas de ITU en el año 2012, el cultivo fue negativo en cuatro casos (31\%) de las muestras recogidas al acecho (13 de las 36), y se halló flora mixta o contaminación de la muestra en un paciente (7,7\%). De manera similar, en aquellas muestras recogidas mediante sondaje vesical (13 de las 36 sospechas de ITU), el urocultivo fue negativo en cinco casos (38,5\%), y en ninguna 
muestra se halló flora mixta. También hubo cinco pacientes en los que la muestra para urocultivo fue recogida con bolsa; en todos los casos este fue positivo. Siendo equivalente el acecho en niños no continentes y la micción media en adultos, las cifras de muestras contaminadas en esta población se sitúan alrededor de esta cifra. En un estudio multicéntrico realizado por Bekeris et al. analizando las tasas de contaminación en 127 laboratorios, hablan de una frecuencia de hasta el $41,7 \%^{16}$. Cabedo García et al. logran reducir la tasa de contaminaciones del 56\% al 41\% mejorando la técnica de recogida por media micción ${ }^{17}$.

\section{CONCLUSIONES}

Resulta fundamental en Urgencias de Pediatría disponer de un método de recogida de orina sensible, específico, sencillo y poco invasivo para los pacientes no continentes.

La recogida de orina mediante acecho supone un método incruento y fácil de llevar a cabo en este grupo de pacientes, si bien se debe tener en cuenta la necesidad de colaboración por parte de la familia y la utilización de una técnica de recogida estandarizada. Asimismo, la recogida de orina mediante acecho reduce el tiempo de espera en

\section{BIBLIOGRAFÍA}

1. Grupo de trabajo de la Guía de Práctica Clínica sobre Infección del Tracto Urinario en la Población Pediátrica. Guía de Práctica Clínica sobre Infección del Tracto Urinario en la Población Pediátrica. Plan de Calidad para el Sistema Nacional de Salud del Ministerio de Sanidad, Política Social e Igualdad. Instituto Aragonés de Ciencias de la Salud; 2011. Guías de Práctica Clínica en el SNS: I+CS No 2009/01. Disponible en www.guiasalud.es/egpc/ITU/comple ta/index.html

2. Romero FJ, Barrio AR. Punción suprapúbica y sondaje vesical. An Pediatr Contin. 2003;1:97-100.
Urgencias al ser más fiable que la recogida mediante bolsa adhesiva.

La bolsa adhesiva es un método que requiere confirmación en los casos positivos, con frecuencia mediante sondaje vesical. El acecho permite la recogida de orina sin precisar confirmación, evitando así el sondaje y los riesgos derivados de este.

No se ha objetivado un aumento de diagnósticos falsos de ITU en los pacientes con muestra recogida por el acecho en comparación con los recogidos por bolsa adhesiva con o sin confirmación por sondaje vesical.

Por tanto el acecho supone una alternativa, equivalente al chorro miccional medio en pacientes continentes, que permite una recogida de orina fácil, incruenta, rápida, sin precisar confirmación y válida en pacientes no continentes con síndrome febril sin foco o sospecha de ITU.

\section{CONFLICTO DE INTERESES}

Los autores declaran no presentar conflictos de intereses en relación con la preparación y publicación de este artículo.

\section{ABREVIATURAS}

IMIP: Institute of Maternal and Child Health of Pernambuco; - ITU: infección del tracto urinario; • L/c: leucocitos por campo.

3. Craig JC. Urinary tract infection: new perspectives on a common disease. Curr Opin Infect Dis. 2001; 14:309-13.

4. American Academy of Pediatrics. Urinary tract infection: clinical practice guideline for the diagnosis and management of the initial UTI in febrile infants and children 2 to 24 months. Pediatrics. 2011;128: 595-610.

5. Dubos F, Raymond J. Pyélonéphrite aiguë du nourrisson: stratégies diagnostiques. Archiv Pédiatr. 2012;19:S101-8.

6. Al-Orifi F, McGillivray D, Tange S, KramerMS. Urine culture from bag specimens in young children: are the risks too high? J Pediatr. 2000;137:221-6. 
7. Grisaru-Soen G, Goldman R, Barzilai A, Lotan D, Keller N. False-positive urine cultures using bag collection. Clin Pediatr (Philadelphia). 2000;39:499-500.

8. Hernangómez Vázquez S, Oñoro G, de la Torre Espí M, Martín Díaz MJ, Novoa-Carballal R, Molina Cabañero JC. Complicaciones del cateterismo vesical realizado en un servicio de urgencias para obtener una muestra de orina. An Pediatr (Barc). 2011;75:253-8.

9. Karacan C, Erkek N, Senel S, Akin Gunduz S, Catli G, Tavil B. Evaluation of Urine Collection Methods for the Diagnosis of Urinary Tract Infection in Children. Med Princ Pract. 2010;19:188-91.

10. Alam MT, Coulter JB, Pacheco J, Correia JB, Ribeiro MG, Coelho MF, et al. Comparison of urine contamination rates using three different methods of collection: clean-catch, cotton wool pad and urine bag Ann Trop Paediatr. 2005;25:29-34.

11. Tosif S, Baker A, Oakley E, Donath S, Babl FE. Contamination rates of different urine collection methods for the diagnosis of urinary tract infections in young children: an observational cohort study. J Paediatr Child Health. 2012;48:659-64.

12. González Rodríguez JD, Rodríguez Fernández LM. Infección de vías urinarias en la infancia. Protoc Diagn Ter Pediatr. 2014;1:91-108.
13. Ochoa Sangrador C, Málaga Guerrero S; Panel de Expertos de la Conferencia de Consenso; Grupo Investigador de la Conferencia de Consenso. Recomendaciones de la Conferencia de Consenso "Manejo Diagnóstico y Terapéutico de las Infecciones del Tracto Urinario en la Infancia”. An Pediatr (Barc). 2007;67:517-25.

14. Urinary tract infection in children Diagnosis, treatment and long-term management. En: NICE [en línea] [consultado el 28/08/2015]. Disponible en www.nice.org.uk/guidance/cg54/resources/guidan ce-urinary-tract-infection-in-children-pdf

15. Herreros Fernández ML, González Merino N, Tagarro García A, Pérez Seoane B, de la Serna Martínez M, Contreras Abad MT, et al. A new technique for fast and safe collection of urine in newborns. Arch Dis Child. 2013;98:27-9.

16. Bekeris LG, Jones BA, Walsh MK, Wagar EA. Urine culture contamination. A College of American Pathologists Q-Probes study of 127 laboratories. Arch Pathol Lab Med. 2008;132:913-7.

17. Cabedo García VR, Novoa Gómez C, Tirado Balaguer MD, Rodríguez Morquecho N, Rodríguez Bailo MT, Solá Sandtner A. ¿Es importante la técnica de recogida de la orina para evitar la contaminación de las muestras? Aten Primaria. 2004;33:140-4. 